\title{
EchoGéo
}

$56 \mid 2021$

Les dynamiques spatiales contemporaines de la Russie

\section{A qui appartient la mémoire des gares? Un manifeste pour une géopoétique engagée des infrastructures}

Entretien avec Gilles Rabin réalisé par Nacima Baron le 17 avril 2021 à Paris

\section{Gilles Rabin et Nacima Baron}

\section{OpenEdition}

Journals

Édition électronique

URL : https://journals.openedition.org/echogeo/21513

DOI : $10.4000 /$ echogeo. 21513

ISSN : 1963-1197

Éditeur

Pôle de recherche pour l'organisation et la diffusion de l'information géographique (CNRS UMR 8586)

Référence électronique

Gilles Rabin et Nacima Baron, «A qui appartient la mémoire des gares? Un manifeste pour une géopoétique engagée des infrastructures », EchoGéo [En ligne], 56 | 2021, mis en ligne le 25 juin 2021, consulté le 31 juillet 2021. URL : http://journals.openedition.org/echogeo/21513; DOI : https://doi.org/ $10.4000 /$ echogeo. 21513

Ce document a été généré automatiquement le 31 juillet 2021.

EchoGéo est mis à disposition selon les termes de la licence Creative Commons Attribution - Pas d'Utilisation Commerciale - Pas de Modification 4.0 International (CC BY-NC-ND) 


\section{A qui appartient la mémoire des gares? Un manifeste pour une géopoétique engagée des infrastructures}

Entretien avec Gilles Rabin réalisé par Nacima Baron le 17 avril 2021 à Paris

Gilles Rabin et Nacima Baron

\section{RÉFÉRENCE}

Gilles Rabin, préface de Pascale Laborier Berlin est une gare

2021. Elya Editions, $137 \mathrm{p}$. 


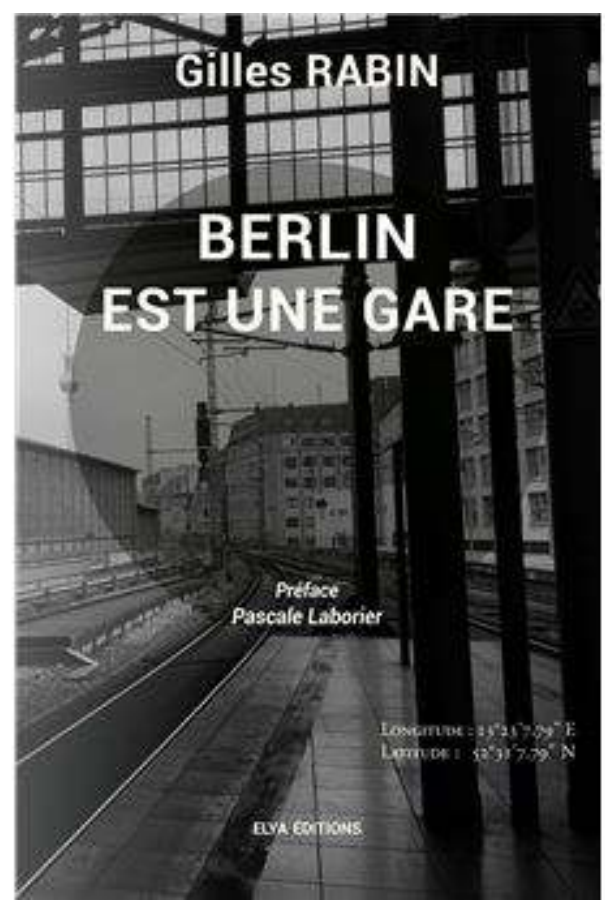

Nacima Baron (NB) : Cher Gilles, le titre de votre ouvrage paru en 2021 aux Éditions Alya interpelle les géographes. II sonne comme un jugement de fait. Mais derrière l'expression d'une évidence irréfutable, on décèle deux énigmes.

La première provient de votre fascination pour les lieux de transport, et notamment pour l'univers ferroviaire. A ce titre, ce livre n'est pas votre coup d'essai (Nègre et al., 2012). Mais au-delà du magnétisme que chacun peut - ou pas - ressentir à l'égard des gares, vous tentez de vous rapprocher d'éclaircir, pour vous d'abord, pour vos lecteurs aussi bien sûr, la question suivante : en quoi une gare peut-elle raconter une ville, jusqu'à quel point peut-elle en aspirer et en exprimer l'identité profonde? Est-ce bien cela le projet de cet ouvrage?

Gilles Rabin (GR). Chère Nacima, vous savez, je ne suis pas un scientifique. Certes je suis docteur d'économie, mais mon rapport au territoire s'est construit pendant plus de quatre décennies - j'ai soixante ans - à partir d'un travail de conseil et d'intervention auprès des acteurs urbains, en France, en Allemagne et au-delà. Je fréquente cette gare et son quartier lieu depuis que j'ai 17 ans. Je m'y suis rendu des centaines de fois.

Dans ce livre, j'ai voulu expliquer que la construction matérielle de la forme urbaine aussi bien que l'élaboration des idées et des images qui forment la mémoire vive d'une ville - entretiennent des rapports à la fois très puissants et très enchevêtrés avec une infrastructure aussi massive que des lignes de chemin de fer et des gares. À partir du cas évidemment très particulier de Berlin, je montre l'intérêt d'interroger l'infrastructure de transport urbain du point de vue historique et mémoriel, urbain et social, culturel et psychologique. Pour moi, la grande gare est comme la cathédrale $\mathrm{du} 19^{\mathrm{e}}$ siècle. Elle permet de remonter le temps et de saisir ce qui fait l'âme d'une ville à travers trois dimensions. La gare de Friedrichstrasse nous introduit d'abord à ce qui persiste dans le temps de la ville, à la résistance des lieux, des formes et des symboles. Ensuite, cette gare unique révèle aussi ce qui rend Berlin unique parmi les villes, comme toutes les villes qui sont des objets jamais sériels, toujours singuliers. Enfin, l'excavation dans le passé de la gare et de la ville fait aussi accéder à une certaine rémanence (ce qui reste quand tout change), et c'est aussi un sujet car Berlin est l'une 
des villes d'Europe qui a connu la plus grande frénésie de reconstructions depuis la chute du Mur et le retour des institutions de l'État allemand.

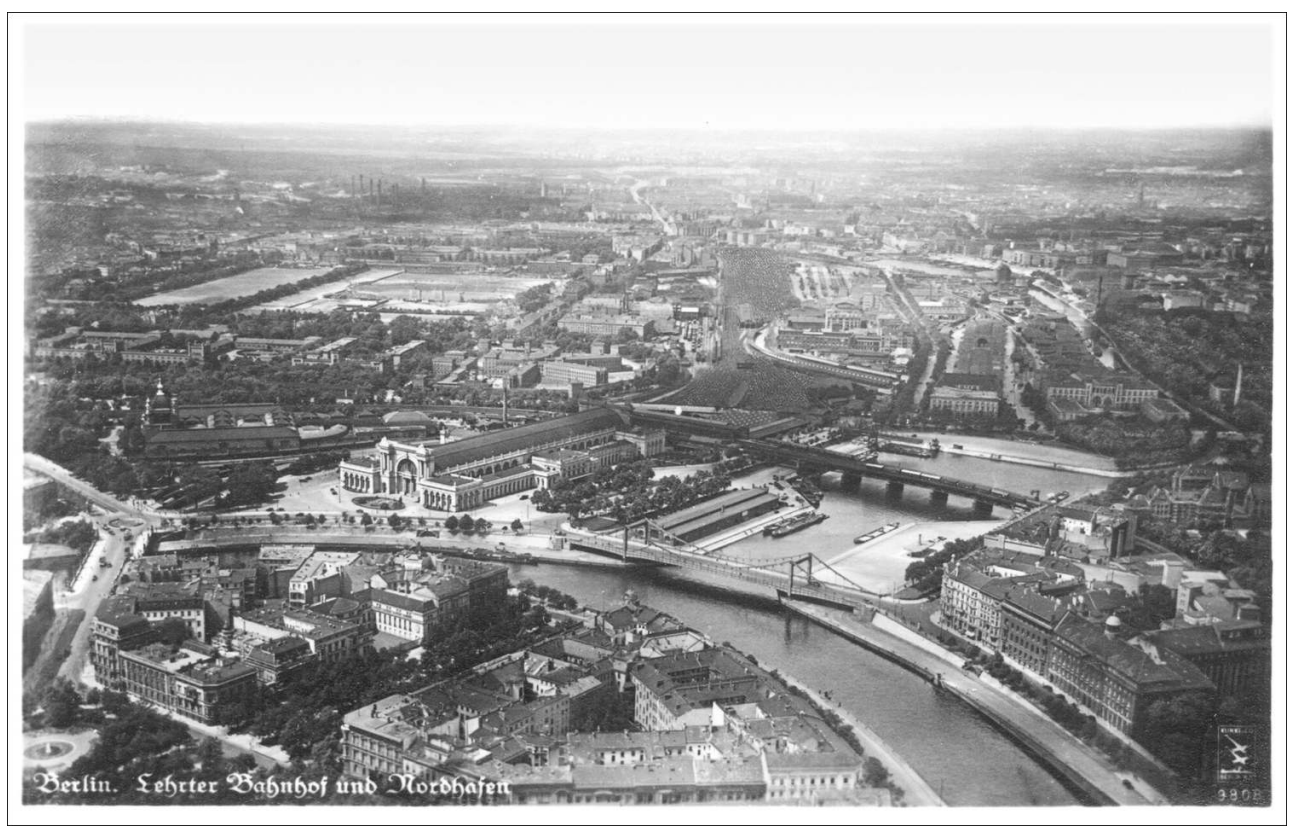

Source : Bundesarchv

NB. Parlons un peu de votre méthode de travail avant de plonger dans l'extraordinaire richesse urbanistique, sociale et architecturale de l'avenue et du quartier de la gare Friedrichstrasse. Votre livre est autant une invitation à la déambulation, sinon à la dérive urbaine, que le résultat d'un véritable travail d'érudition. La précision des informations, sur le passé aussi bien que sur le présent (je pense par exemple à votre relevé systématique des enseignes alimentaires de la gare actuelle) montre l'importance de la compilation documentaire et la conduite de véritables enquêtes de terrain. Quelle sont vos pratiques de l'espace et de la recherche urbaine?

GR [Gilles Rabin se lève et prend dans sa bibliothèque un atlas ferroviaire de l'Allemagne qu'il ouvre à la page de Berlin]. Je suis d'abord un promeneur, un passant qui s'intéresse à la résonnance intime de l'espace, comme le rappelle également une de mes autres expériences de marche urbaine (Rabin et Gwazdzinski, 2007). Ma méthode s'appuie sur le cheminement pédestre. La gare est partie prenante d'une avenue de 3,3 km de long que j'ai sillonnée en tous sens, et donc j'ai étudié chacun des bâtiments dans son histoire, dans son devenir morphologique et fonctionnel. Ma méthode est aussi scopique. Elle consiste aussi à s'asseoir sur un banc, en face du portail monumental de la gare par exemple, et à observer toutes celles et ceux qui bougent (les voyageurs, les passants) comme celles et ceux qui ne bougent pas (les clients à la terrasse de cafés, les sans domicile fixe). Sur le plan du résultat, c'est vrai que le livre fait écho à la géographie culturelle de Denis Cosgrove ${ }^{1}$ mais il peut aussi intéresser des chercheurs tournés vers la géographie sociale car il introduit à des réflexions autour de la gentrification des quartiers de gare, de la commodification des lieux de transport, à une réflexion sur la place des migrants dans la ville aussi. 


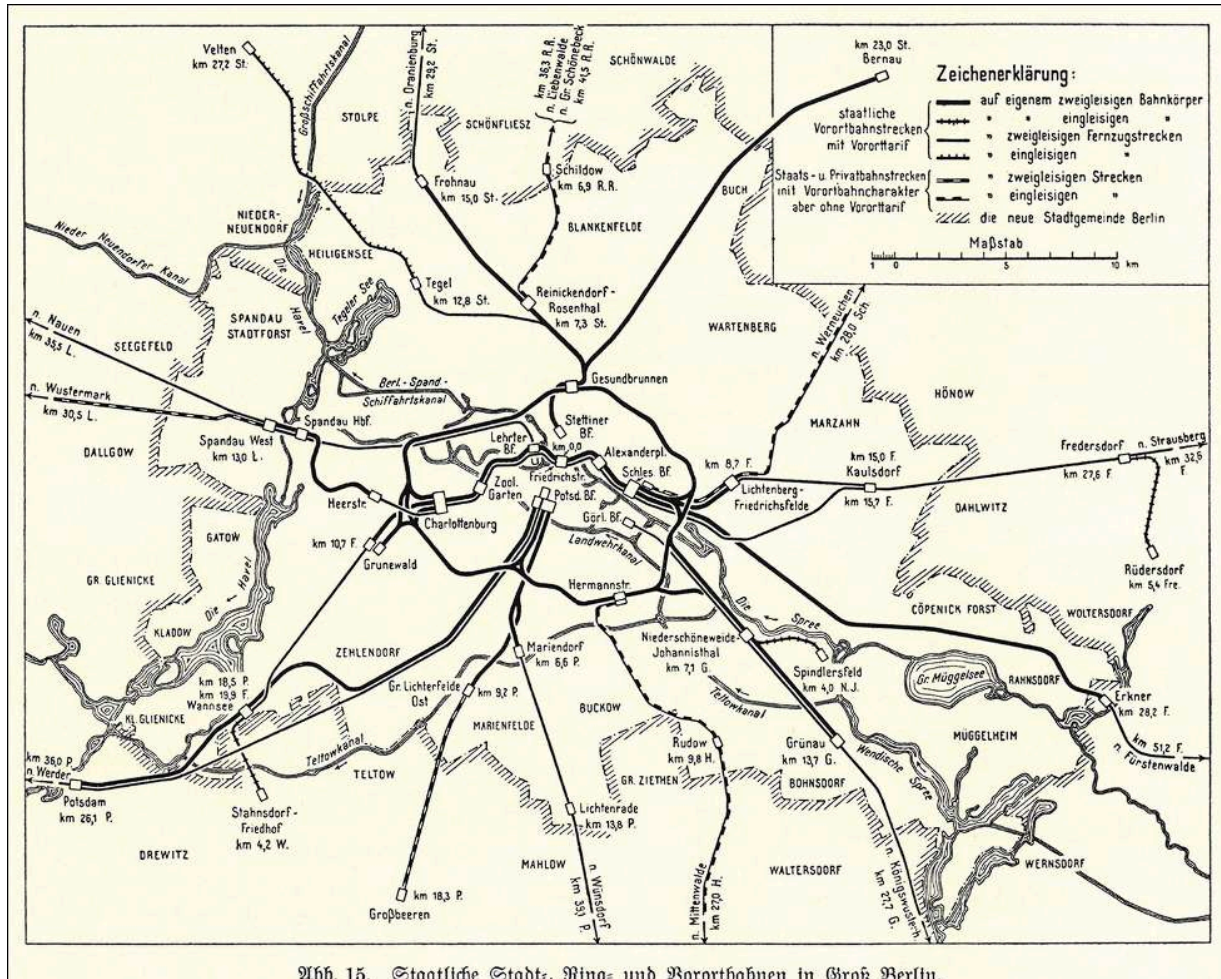

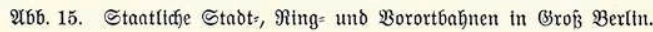

Source: Bundesarchv.

NB. Venons-en au cœur de l'analyse. Ce qui fait que la gare de Friedrichstrasse vous " parle », c'est qu'elle permet de revisiter cinq épisodes d'histoire urbaine : le Berlin baroque de l'empereur Frédéric, le moment prussien, l'entre-deux guerres, le Berlin divisé de la guerre froide et la période contemporaine, qu'on peut caractériser comme une étape de normalisation dont un travail de psychogéographie a déjà montré le caractère tout à fait singulier (Grésillon, 2017).

GR. Oui, bien sûr, l'empreinte de la durée temporelle dans l'espace est au cœur de mon travail. La gare a le même nom que l'avenue, elle-même dessinée au temps de Frédéric II (1712-1786) dans la perspective de l'urbanisme des Lumières. L'axe s'est encore structuré tout au long du 19E siècle et la gare a été construite en 1882. Elle est inaugurée par le chancelier Bismarck. Le dessin du réseau ferroviaire est au service des desseins du régime : faire de Berlin la capitale d'un territoire centralisé, utiliser le réseau ferré comme l'outil de cette domination politique et économique. Vient ensuite la période de l'érection des bâtiments emblématiques (hôtels, cafés, théâtres, studios de cinéma, etc.) qui affirment la réalité métropolitaine mais qui sous-tendent aussi l'effervescence culturelle du Berlin des années 1930. Une génération plus tard, la gare est aussi le théâtre d'autres réalités géopolitiques. Elle constitue l'unique point de passage (avec Check Point Charlie à quelques centaines de mètres plus loin) entre Berlin est et Berlin ouest. Enfin, depuis la chute du Mur, beaucoup de ces traces ont été ou sont effacées. Des immeubles de bureaux de style international et des grands magasins (par exemple celui de Jean Nouvel pour les Galeries Lafayette) effacent des empreintes du Berlin du long vingtième siècle. Je partage la nostalgie ou plutôt l'« ostalgie » des Berlinois de l'ancienne zone sise en RDA, car cette souffrance n'est pas entendue. Je dirais même qu'elle est un tabou, on n'a pas le droit, je ne dirai pas de regretter une période d'oppression, mais d'exprimer l'intérêt pour les traces 
physiques que cette époque a laissée. En ce sens, la gare Friedrichstrasse constitue un des seuls marqueurs du temps long de la ville, avec quelques autres grandes infrastructures comme l'aéroport de Tempelhof. Persistance, permanence, survivance, résistance, rémanence... Il y a tant de choses qui « persévèrent dans leur être » à Berlin, dans les corps, dans les têtes, comme dans les plis de l'espace urbain, et j'ai voulu comprendre le rôle joué par la gare dans ce contexte.

NB. Il est en effet très intéressant de comprendre comment vous articulez les métriques et les échelles spatiales, Votre livre parle tout ensemble du destin géographique de Berlin, de ses rapports avec l'Allemagne du nord mais aussi avec les régions de l'ouest, de l'est et du sud de l'Europe d'où sont venues nombre de populations migrantes qui ont fréquenté l'avenue, ou qui y sont enterrées non loin (je pense au cimetière où reposent des Huguenots) et qui fréquentent encore l'avenue. Vous travaillez donc sur un quartier, sur une longue avenue et ses axes adjacents, dont les carrefours sont des pivots majeurs, vous parcourez la gare dans tous ses étages et les couloirs du métro, vous tournez autour (de part et d'autre de la Sprée), vous vous arrêtez sur des bâtiments individuels et puis sur des micro-objets : statues du souvenir des enfants déportés, bancs, affiches ...

GR. Bien entendu, car j'associe la réalité de la matière urbaine (Hocquet et al., 2017) de Berlin avec la profusion des récits, des imaginaires, sinon des légendes (Morel, 2014) de la ville. J'associe donc tous ces régimes de spatialité, qu'elle soit leur dimension ou leur nature. Je réfléchis à partir de différents types d'axes (la lignes ferrées, l'artère commerçante de Friedrichstrasse), je m'arrête sur des nœuds (le pôle d'échange entre le métro et le train régional dans la gare, avec ses corridors et escaliers, sa librairie ouverte même la nuit, j'explore les carrefours de l'avenue avec des axes secondaires). L'approche zonale est aussi importante: le grand paysage autant que l'échelle métrique m'offrent des prises pour montrer les continuités et les ruptures qui font de cette gare élargie à son quartier une sorte de "point vélique» de la civilisation. Toutes les forces de chaque époque se concentrent, comme le vent sur les voiles, à Friedrichstrasse, et en font une sorte d'île. Une autre clé de lecture de la spatialité de la gare est l'opposition dedans-dehors. C'est très étonnant d'ailleurs de voir le contraste entre l'intérieur de la gare, où tout a changé avec le temps (on y trouve les panneaux digitaux d'information sur les trains et des restaurants rapides sans âme) et l'extérieur, c'est-à-dire la façade et plus généralement l'enveloppe externe du bâtiment. Le langage stylistique de l'architecte Johannes Volmer cristallise ce mariage entre la modernité du rail, la volonté de faire capitale et donc d'imprimer une impression d'un bâtiment « Kolossal », et d'adhérer à la modernité. 
Illustration 3 - Les statues Train to life du sculpteur Frank Meisler

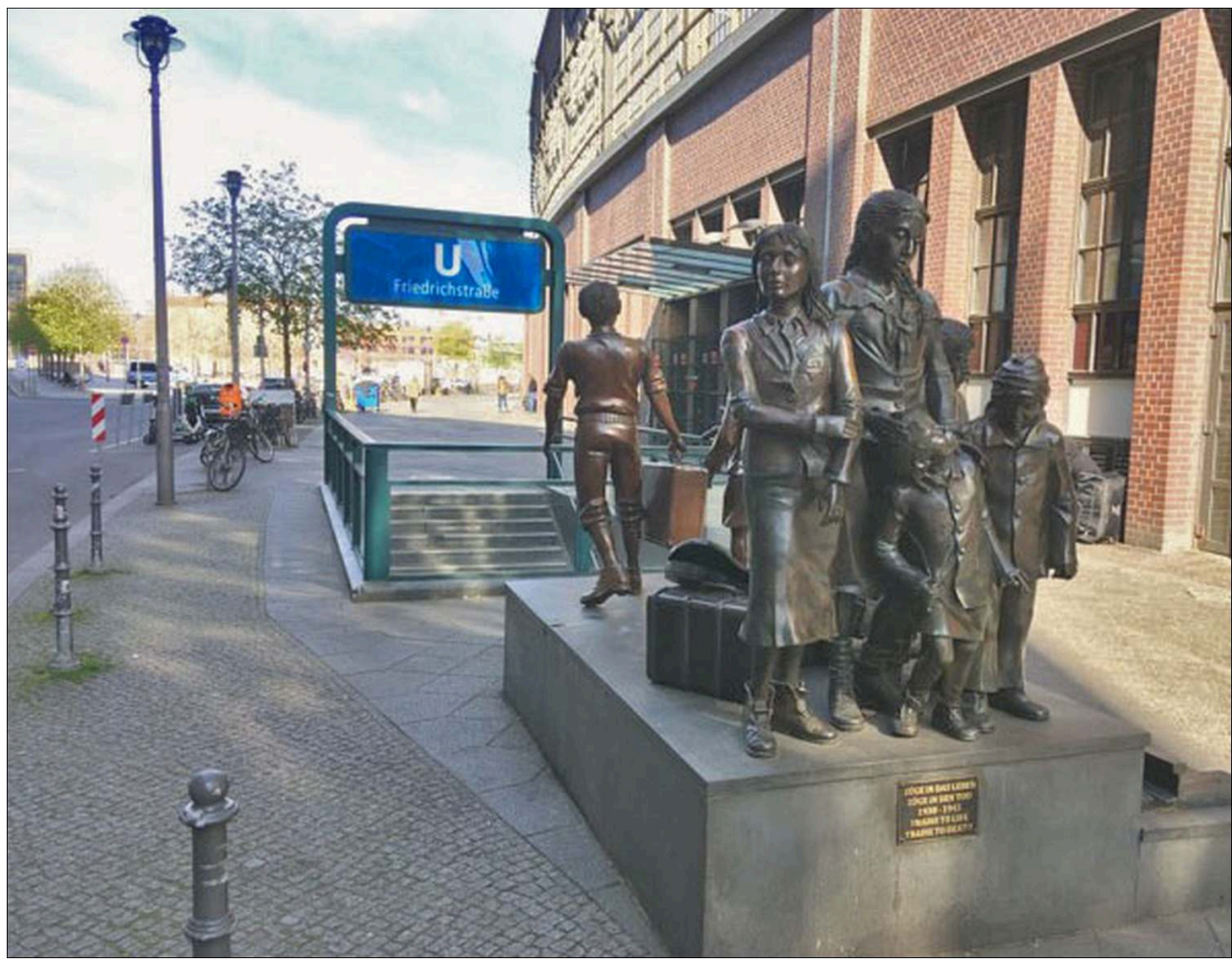

Ces statuts rappellent les groupes d'enfants envoyés aux camps d'extermination d'un côté, et les enfants sauvés par leur envoi au Royaume-Uni de l'autre.

Source : Manuel Tonneau (libre de droits).

NB. Nous allons justement revenir à la manière dont la gare et l'avenue de Friedrichstrasse non seulement font lieu mais racontent la production de quelques-uns des hauts-lieux (Flier, 2017) qui marquent indéfectiblement la culture allemande. Arrêtons-nous sur BerlinBabylone des années 1920.

GR. Prenons un instant dans la vie de l'avenue. Nous sommes le 31 août 1928. Bertold Brecht célèbre la première de l'Opéra de Quat' Sous dans un théâtre du quartier Mitte qui deviendra plus tard, le Berliner Ensemble. C'est un soir d'été où une foule considérable se croise au niveau du café Kranzler qui marque le carrefour de Friedrichstrasse avec l'avenue Unter den Linden (ce carrefour voit déjà passer, à cette époque, plus de 120000 piétons et 13000 voitures par jour). Des militaires qui habitent les casernes d'artillerie au nord de l'avenue l'ont descendue en groupe, attirés par les cabarets, les lumières des grands magasins, les lupanars. Ils retrouvent les prostituées qui, après avoir chanté sur la scène de théâtres comme Am Zirkus et peut-être croisé une Marlene Dietrich débutante, sont « descendues de la scène ». Ces femmes cherchent aussi la compagnie des riches étrangers de l'ouest qui habitent le Central Hotel ou d'autres établissements de luxe. Récemment installées dans les quartiers pauvres de l'est (comme les serveurs des restaurants et comme les techniciens qui font tourner les studios de cinéma, dont celui des frères Lumière au sud de l'avenue), ce nouveau prolétariat berlinois, souvent venu de Pologne, d'Ukraine, est arrivé sur l'avenue Friedrichstrasse, grâce au métro souterrain (en service depuis 1923) et à la gare (celle-ci accueille déjà 50000 passagers par jour, soit 16 millions de passagers par an. Georg Simmel (Jonas, 2008) est mort depuis dix ans déjà, mais son bureau à l'Université est voisin de l'avenue. Il a lui aussi peut-être écrit 
son livre Les grandes villes et la vie de l'esprit en observant à la terrasse de la Friedrichstrasse l'étonnante diversité du brassage social et ethnique, la vitalité des échanges et l'intensité du trafic à la terrasse du Berlin de ces années rugissantes.

$\mathrm{Au}$ fond, je veux dire par là que la gare de Friedrichstrasse constitue à la fois une porte d'entrée et l'un des creusets de la centralité métropolitaine. Je la compare à notre gare parisienne de Saint-Lazare quarante ans plus tôt. Je vois une comparaison possible dans la dynamique de la construction progressive de l'édifice, de plus en plus imposant pour accueillir plus de voies ferrées. Je note que la gare Saint-Lazare, dans les années 1880, associe agencement urbain, stratification sociale et créativité culturelle. À l'époque, le grand hall s'organise avec sa salle d'attente, ses guichets, et la grande salle des pas perdus, où les lycéens de Condorcet organisent de folles courses et fondent le club du Racing : à l'époque, où le sport constitue un élément majeur de distinction de classe. La gare est aussi l'outil de construction métropolitaine, et je fais aussi le rapprochement car la gare urbanise la banlieue (ici, il faut comprendre la banlieue pavillonnaire tout le long de la vallée de la Seine). Je trouve aussi des parallèles dans la manière dont la gare accompagne la structuration d'un quartier rapproché. Elle crée aussi du vide: en particulier un parvis où se pressent les autobus et les taxis. Je note aussi le rôle que joue la gare comme attracteur pour la clientèle des brasseries et des grands magasins et sa fertilité pour l'imaginaire. Ici, je songe à Emile Zola qui écrit La Bête Humaine en écoutant, de sa terrasse de Médan, les trains de Saint-Lazare aller et venir.

NB. Parlons maintenant d'un autre temps fort de l'histoire de Berlin et de cette gare. Du 3 avril 1962 au 2 juin 1990, Friedrischtrasse est appelée la gare des pleurs. Elle est utilisée pour le transit entre l'est et l'ouest de la ville. Comme plus de 5 milions et demi de Berlinois entre 1963 et 1966, vous avez maintes fois emprunté le Kontrolpassierpunkt et les fameux quais $\mathrm{B}$ et $\mathrm{C}$ qui permettaient d'accéder aux trains... dont vous notez qu'aucun conducteur (la gestion de ces trains étant assurée par la RDA) n'a tenté de passer à l'ouest. Vous relatez ce que la division a fait à la gare, dans l'espace et dans les usages : réorganisation technique de l'édifice, gestion policière sourcilleuse des circulations, portée émotionnelle de ce rite de passage (Burdy, 2004).

GR. Je me souviens exactement de ces trains venant de l'est, qui après Ostbahnhof, roulent $1,3 \mathrm{~km}$ ni trop vite ni trop lentement pour éviter que des voyageurs ne sautent en marche. Je me souviens des tours de surveillance de la Volkspolizei et du bâtiment, aujourd'hui détruit, au long duquel les passagers traversent un couloir et se prêtent aux formalités (vérification d'identité, contrôle des bagages, paiement des 25 marks de droit de passage). La sécurisation va de pair - c'est un peu comme aujourd'hui du reste - avec la multiplication des commerces et l'encouragement à la consommation. C'est en cela que la gare et le quartier se transforment des années 1960 aux années 1980. La gare fonctionne comme une cash machine pour le régime de RDA. Le pouvoir la remplit de ces Intershop où les Allemands de l'ouest sont invités à laisser leurs marks. Les architectes du régime (notamment Manfred Prasser et Ehrhardt Gißke) aménagent des Valuta-Hotels (par exemple le Dom Hotel devenu le Hilton et l'hôtel de la place de l'Académie). Les visiteurs de l'ouest y sont hébergés contre la monnaie forte. Loin d'ignorer le bâti historique qui a survécu aux bombardements des années 1940, ils le restaurent : ainsi, l'église française bâtie par et pour les Huguenots en 1699 est rénovée en 1983. Plus tard, le régime d'Honecker accentue cette démarche et ses architectes dessinent un programme urbanistique associant hôtels, salles de séminaires, magasins de luxe, espaces de loisir ... Les 
arcades de Friedrichstrasse témoignent-elles, comme une publication le suggère (Urban, 2008), du fait qu'un certain nombre de traits de l'idéologie du capitalisme urbain (consommation de masse, acceptation de l'inégalité sociale, réinterprétation architecturale du patrimoine stylistique impérial dans les bâtiments modernes plutôt qu'immeubles de béton de l'architecture socialiste...) ont pénétré les consciences des dirigeants? Je dirais plutôt pour ma part que le besoin éperdu de ressources financières du pouvoir de RDA oriente la gare et son quartier dans une logique de production de valeur. La gare représente dès lors un asset. De ce point de vue, l'approche contemporaine de la Deutsche Bahn n'est finalement pas très différente de celle de la fin de l'époque RDA.

Illustration 4 - Aspect contemporain de la gare de Friedrichstrasse

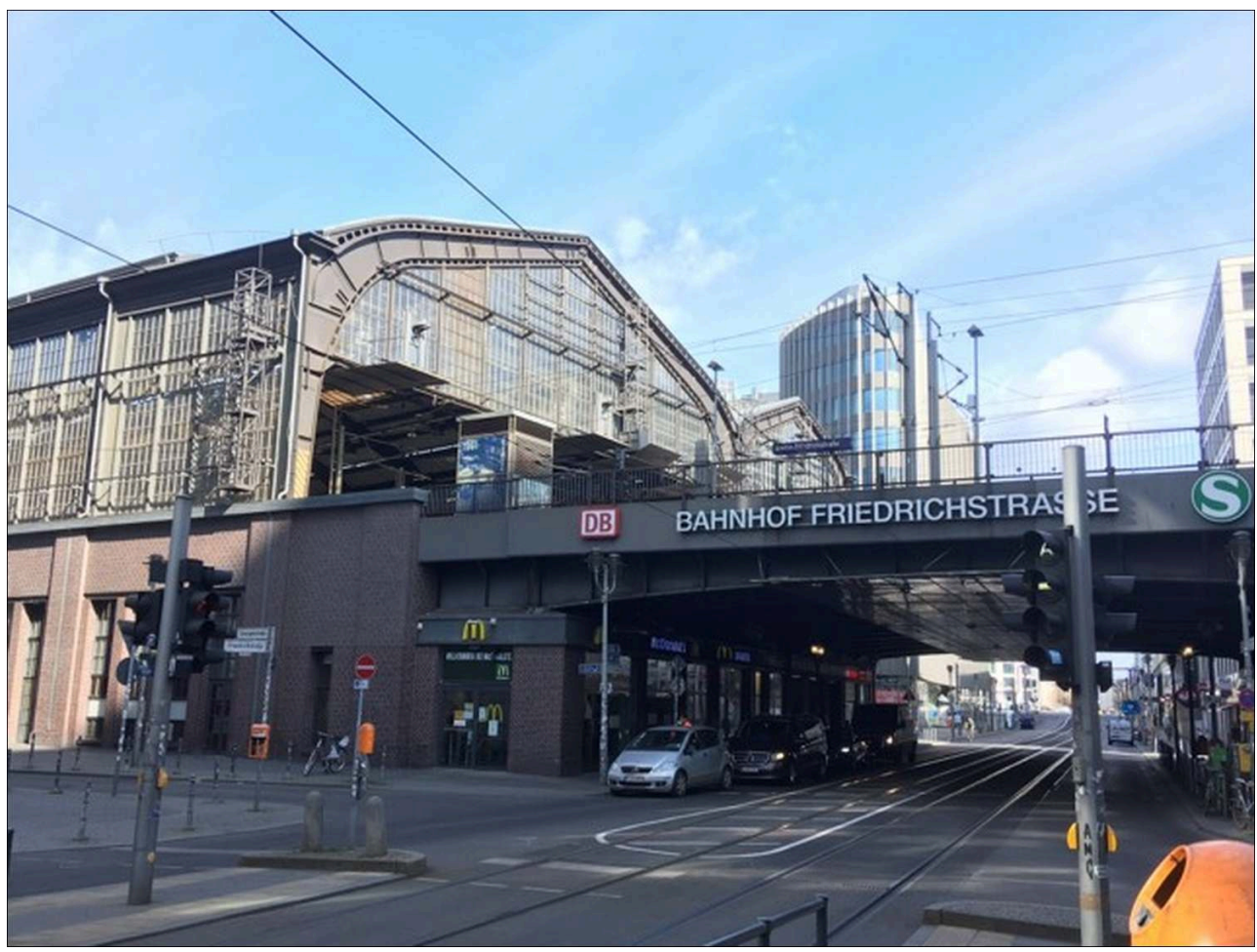

Source : Manuel Tonneau (libre de droits).

NB. Passons maintenant à la période de la réunification. C'est la phase au cours de laquelle des «starchitectes » comme Jean Nouvel, I.M. Pei et Oswald Mathias Ungers dessinent le long de Friedrichstrasse de nouveaux bâtiments dont le cachet néohistorique voisine avec le verre et l'acier. Une nouvelle foule remplace celle des années 1930 sur les trottoirs de l'avenue: cadres financiers et ingénieurs de l'industrie digitale, étudiants de tous pays inscrits à l'Université Humboldt toute proche, fonctionnaires rejoignant les bureaux des institutions du gouvernement allemand transféré de Bonn au bord de la Sprée. Il y a un certain retour en grâce des cabarets, théâtres et lieux de plaisir sur l'avenue. Mais vous avez également des mots assez durs pour caractériser la manière dont Deutsche Bahn réorganise le système de transports métropolitain et la hiérarchie des gares dans les années 1990 (Rousseau et Guihéry, 2017) de manière à faire de l'ancienne Lerhterbahnhof la nouvelle gare centrale. Le contexte de la préparation de la coupe du monde de foot de 2006 autorise bien des mégaprojets. A partir de 2006, justement, la gare Friedrichstrasse 
se voit rétrograder au rang de gare secondaire, et ne garde plus que les circulations régionales ou S Bahn...

GR. Berlin Est présente à partir de la réunification beaucoup de parcelles vastes et vides, d'un coût très inférieur aux cours du marché foncier à l'ouest. Il est compréhensible qu'elles soient donc très rapidement convoitées puis remplies, tandis que la démolition - reconstruction bat aussi son plein et que tant d'immeubles de l'avenue soient remplacés. Le quartier est donc transformé au prix de nombreuses controverses (Grésillon, 2003). Pourtant, l'esprit de Friedrichstrasse ne disparait pas complètement. Ce lieu ne s'est pas aseptisé tant que cela. Le quartier attire les jeunes d'abord à cause de sa très grande accessibilité depuis le reste de l'agglomération, ensuite parce qu'il reste quelque chose d'impalpable, mais d'historique et d'authentique. Le débit mythique de bière bavaroise qu'affectionnaient les fonctionnaires de Bonn et qui a accompagné leur relocalisation administrative à Berlin s'est installé justement devant la gare de Friedrichtrasse, et pas devant la gare centrale. Depuis les quais de la gare Friedrichstrasse, on touche presque du doigt la tour de télévision, qui est aussi un peu l'emblème de la ville ... le site garde donc de la densité et de l'intensité sociale et historique.

NB. Vous nous dites que c'est à travers la gare qu'une ville négocie sa relation entre passé et présent. Mais en quoi s'y joue-t-il également son rapport avec le futur? Comment voyezvous l'avenir des récits urbains de Berlin?

1 GR. D'un côté, évidemment, toutes les villes sont engagées dans des démarches d'urban branding et Berlin ne fait pas exception. Cependant, ce discours publicitaire évolue : l'image de la scène alternative berlinoise, des immenses boîtes de nuit, de la musique techno et des squats est un peu derrière nous. En revanche, ce qui ne change pas est la nature cosmopolite de cette ville. Berlin a été et reste une ville-monde. Les gares depuis Georg Simmel justement - ont beaucoup à nous montrer sur disent la qualité des interactions que les individus d'une société entretiennent les uns envers les autres. Pensez à la gare de Munich qui a accueilli 63000 réfugiés syriens, en deux semaines à l'automne 2015. Ces réfugiés, les habitants sont venus les saluer, les applaudir, ils ont offert des nounours aux enfants à la descente du train. Observateur des nombreuses gares de Berlin aujourd'hui, je repère combien ce sont des lieux qui attirent les primomigrants, aujourd'hui afghans, tchétchènes, moldaves, entre autres. Ce sont des lieux ouverts et vastes, fortement fréquentés (certes moins depuis la COVID) dans lesquels ces derniers ont envie de se fondre dans la masse. Les villes d'aujourd'hui ont grand besoin de ces espaces d'ouverture qui, par la vacuité qu'ils offrent, créent du commun. Trop d'organisation et de fonctionnalisme dans les gares aujourd'hui (" tiers lieux » et autres espaces dédiés) tue la capacité, pour l'usager, de ressentir la qualité du lieu et de s'approprier, singulièrement ou collectivement, l'espace. Dans la gare, c'est le lieu qui impose, non la logique dite des $3 \mathrm{~S}$ (service, sécurité et propreté) qui forment aujourd'hui le pilier du programme de gestion des gares de la Deutsche Bahn, et qui les rendent si monotones. 


\section{BIBLIOGRAPHIE}

Burdy J.-P.., 2004. La liberté coûte un ticket de métro, Transports et circulation dans le Berlin de la guerre froide. Histoire Urbaine, vol. 3, n 11, p. 81-108.

Dury E., 2003. Berlin ou l'expérience des limites. Travaux de l'Institut de Géographie de Reims, $\mathrm{n}^{\circ} 113-114$, p. 53-67.

Flier B., 2017. Le centre-ville de Berlin-Est : entre Porte de Brandebourg et Alexanderplatz : histoire, pouvoir, symboles, enjeux. Allemagne d'aujourd'hui, vol. 3, n² 221, p. 155-161.

Grésillon B., 2003. Les hauts-lieux berlinois : une réappropriation problématique. Les Temps modernes, vol. 4, n 625, p. 119-127.

Grésillon B., 2017. Berlin sur la voie de la normalisation, Essai de psychogéographie. Allemagne d'aujourd'hui, vol. 3, n² 221, p. 39-56.

Hocquet M.., Garrido C., von Hirschhausen B., 2017. Berlin par-delà les ruptures : vivre, raconter et produire les matières de la ville. L'Espace géographique, vol. 46, n² 2, p. 158-173.

Jonas S., 2008, Simmel et Berlin, de la ville à la métropole. In Füzéssery S., Simay P. (dir.), Le choc des métropoles - Simmel, Kracauer, Benjamin et la Großstadt. Éditions de l'Éclat, p. 53-80.

Morel O., 2014. Berlin légendes ou la mémoire des décombres, une capitale littéraire en rêveries et conversations. Presses Universitaires de Vincennes, $230 \mathrm{p}$.

Nègre L., Soulage B., Rabin G., 2012. Quel rail après 2012. Éditions de l'Aube.

Rabin G., Gwazdzinski L., 2007. Périphéries, un voyage à pied autour de Paris. Éditions L'Harmattan Rousseau M.-L., Guihéry L., 2017. Infrastructures et politiques de transport à Berlin : quelle dynamique métropolitaine? Allemagne d'aujourd'hui, vol. 3, n² 221, p. 74-86.

Urban F., 2008. Friedrichstraße, 1987: Neo-historical urban design in the German Democratic Republic. Planning Perspectives, vol. 23, $n^{\circ}$ 1, p. 1-28.

\section{NOTES}

1. Denis Cosgrove (1948-2008) est un des fondateurs de la géographie culturelle. Il a révolutionné l'étude des représentations, l'approche des paysages, la réflexion sur la visualité, le paysage, l'utilisation et la production des cartes. Parmi d'autres grands ouvrages, on peut citer Social formation and symbolic landscape, 1998, University of Wisconsin Press et Geography and vision seeing, imagining and representing the world, 2012, Tauris.

\section{INDEX}

Thèmes : Sur l'Écrit 


\section{AUTEURS}

\section{GILLES RABIN}

Gilles Rabin est directeur de l'innovation des applications et de la science au Centre National d'Etudes Spatiales. Il a notamment publié :

- Rabin G., 2012. Quel rail après 2012. Editions de l'Aube.

- Gwazdzinski L., Rabin G., 2010. Urbi et orbi Paris appartient à la ville et au monde. Nouvelles éditions de l'Aube.

- Rabin G., Gwazdzinski L., 2008. La fin des Maires. Dernier inventaire avant disparition. FYP Editions, Collection Présence. 